\title{
The string BCJ relations revisited and extended recurrence relations of nonrelativistic string scattering amplitudes
}

\author{
Sheng-Hong Lai, Jen-Chi Lee and Yi Yang \\ Department of Electrophysics, National Chiao Tung University, \\ 1001 University Street, Hsinchu, ROC \\ E-mail: xgcj944137@gmail.com, jcclee@cc.nctu.edu.tw, \\ yiyang@mail.nctu.edu.tw
}

ABSTRACT: We review and extend high energy four point string BCJ relations in both the fixed angle and Regge regimes. We then give an explicit proof of four point string BCJ relations for all energy. This calculation provides an alternative proof of the one based on monodromy of integration in string amplitude calculation. In addition, we calculate both $s-t$ and $t-u$ channel nonrelativistic low energy string scattering amplitudes of three tachyons and one higher spin string state at arbitrary mass levels. We discover that the mass and spin dependent nonrelativistic string BCJ relations can be expressed in terms of Gauss hypergeometry functions. As an application, for each fixed mass level $N$, we derive extended recurrence relations among nonrelativistic low energy string scattering amplitudes of string states with different spins and different channels.

Keywords: Scattering Amplitudes, Bosonic Strings, Higher Spin Symmetry

ARXIV EPRINT: 1601.03813 


\section{Contents}

1 Introduction 1

2 Review of high energy string BCJ 3

2.1 Hard string scatterings 3

2.2 Regge string scatterings 5

3 Explicit proof of string BCJ $\quad 8$

4 Nonrelativistic string BCJ and extended recurrence relations $\quad 11$

$\begin{array}{ll}4.1 & \text { Leading trojectory states } \\ & 13\end{array}$

$\begin{array}{lll}4.2 & \text { More general string states } & 15\end{array}$

5 Conclusion $\quad 16$

\section{Introduction}

Inspired by Witten's seminal paper published in 2004 [1], there have been tremendous developments on calculations of higher point and higher loop Yang-Mills and gravity field theory amplitudes. ${ }^{1}$ Many new ideas and techniques have been proposed and suggested on this interesting and important subject. On the other hand, string theory amplitudes have been believed to be closely related to these new results derived in field theory amplitudes. One interesting example was the gauge field theory BCJ relations for color-stripped amplitudes proposed in 2008 [3] and their string origin or the string BCJ relations suggested in 2009 [4, 5]. These field theory BCJ relations can be used to reduce the number of independent $n$-point color-ordered gauge field theory amplitudes from $(n-2)$ !, as suggested by the KK relations $[6,7]$, to $(n-3)$ !.

On the other hand, a less known historically independent development of the "string BCJ relations" was from the string theory side without refering to the field theory BCJ relations. This was the discovery of the four point string BCJ relations in the high energy fixed angle or hard string scattering (HSS) limit in 2006 [8]. Moreover, one can combine these string BCJ relations with the infinite linear relations among HSS amplitudes conjectured by Gross [9-13] and discovered in [14-20] to form the extended linear relations in the HSS limit. For a recent review, see [21]. In constrast to the field theory BCJ relation, these extended linear relations relate HSS amplitudes of string states with different spins and different channels, and can be used to reduce the number of independent HSS amplitudes from $\infty$ down to 1 .

\footnotetext{
${ }^{1}$ See articles in the special issue ref. [2].
} 
Historically, the motivation to probe string BCJ relations in this context was the calculation of closed HSS amplitudes [8] by using the KLT relations [22]. Indeed, it was found [8] that the saddle point calculation of open HSS amplitudes was applicable only for the $t-u$ channel, but not reliable for the $s-t$ channel, neither for the closed HSS amplitude calculation. In addition, it was also pointed out $[8,23]$ that the prefactor $\frac{\sin (\pi u / 2)}{\sin (\pi s / 2)}$ in the string BCJ relations, which was missing in the literature [9-13, 24] for the HSS amplitude calculations, had important physical interpretations. The poles give infinite number of resonances in the string spectrum and zeros give the coherence of string scatterings. These poles and zeros survive in the HSS limit and can not be ignored. Presumably, the prefactor triggers the failure of saddle point calculations mentioned above.

To calculate the closed HSS by KLT relation, one needed to calculate both $s-t$ and $t-u$ channel HSS amplitudes. In constrast to the saddle point method used in the $t-u$ channel, for the $s-t$ channel HSS amplitudes at each fixed mass level $N$, one first used a direct method to calculate the HSS amplitude of the leading trajectory string state, and then extended the result to other string states by using high energy symmetry of string theory or infinite linear relations among HSS amplitudes of different string states at each mass level $N$. As a result, the string BCJ relations in the HSS limit can be derived [8]. All these HSS amplitude calculations for $s-t$ and $t-u$ channels and the related string BCJ relations in the HSS limit were inspired by Gross famous conjectures on high energy symmetries of string theory $[11,12]$, and thus were independent and different from the field theory BCJ motivation discussed above.

In this paper, we will follow up the development on the string theory side of string amplitude calculations. In section II, we will first review the extended linear relations in the HSS limit discussed in [8]. We will also work out the corresponding extended recurrence relations [25, 26] of the Regge string scattering (RSS) amplitudes [27]. Similar to the extended linear relations in the HSS limit discussed above, the extended recurrence relations can be used to reduce the number of independent RSS amplitudes from $\infty$ down to 1 .

We will then give an explicit proof of the string BCJ relations in section III by directly calculating $s-t$ and $t-u$ channel string scattering amplitudes for arbitrary four string states. In constrast to the proof based on monodromy of integration with constraints on the kinematic regime given in [4] without calculating string amplitudes, our explicit string amplitude calculation puts no constraints on the kinematic regime. In section IV, we will calculate the level dependent and the extended recurrence relations of low energy string scattering amplitudes in the nonrelativistic limit. The existence of recurrence relations for low energy nonrelativistic string scattering (NSS) amplitudes come as a surprise from Gross point of view on HSS limit.

In the calculations of low energy extended recurrence relations in section IV, we will take the NSS limit or $\left|\overrightarrow{k_{2}}\right| \ll M_{S}$ limit to calculate the mass level and spin dependent low energy NSS amplitudes. In constrast to the zero slope $\alpha^{\prime}$ limit used in the literature to calculate the massless Yang-Mills couplings $[28,29]$ for superstring and the three point $\varphi^{3}$ scalar field coupling [30-32] for the bosonic string, we found it appropriate to take the nonrelativistic limit in calculating low energy string scattering amplitudes for string states with both higher spins and finite mass gaps. A brief conclusion will be given in section V. 
In the following in section II, we first review historically two independnt developments of the string BCJ relations from field theory and from string theory point of views.

\section{Review of high energy string BCJ}

The four point BCJ relations [3] for Yang-Mills gluon color-stripped scattering amplitudes $A$ were pointed out and calculated in 2008 to be

$$
\begin{aligned}
& t A\left(k_{1}, k_{4}, k_{2}, k_{3}\right)-s A\left(k_{1}, k_{3}, k_{4}, k_{2}\right)=0, \\
& s A\left(k_{1}, k_{2}, k_{3}, k_{4}\right)-u A\left(k_{1}, k_{4}, k_{2}, k_{3}\right)=0, \\
& u A\left(k_{1}, k_{3}, k_{4}, k_{2}\right)-t A\left(k_{1}, k_{2}, k_{3}, k_{4}\right)=0,
\end{aligned}
$$

which relates field theory scattering amplitudes in the $s, t$ and $u$ channels. In the rest of this paper, we will discuss the relation for $s$ and $u$ channel amplitudes only. Other relations can be similarly addressed.

\subsection{Hard string scatterings}

For string theory, in constrast to the field theory BCJ relations, one has to deal with scattering amplitudes of infinite number of higher spin string states. The first "string BCJ relation" discovered was the four point string BCJ relation in the HSS limit [8] worked out in 2006. For the tachyon state, one can express the open string $s-t$ channel amplitude in terms of the $t-u$ channel amplitude [8]

$$
\begin{aligned}
T_{\text {open }}^{(4-\text { tachyon })}(s, t) & =\frac{\Gamma\left(-\frac{s}{2}-1\right) \Gamma\left(-\frac{t}{2}-1\right)}{\Gamma\left(\frac{u}{2}+2\right)} \\
& =\frac{\sin (\pi u / 2) \Gamma\left(-\frac{t}{2}-1\right) \Gamma\left(-\frac{u}{2}-1\right)}{\sin (\pi s / 2)} \frac{\Gamma\left(\frac{s}{2}+2\right)}{\sin (\pi u / 2)} \\
& \equiv \frac{\sin (\pi s / 2)}{\sin (4 \text {-tachyon })}(t, u)
\end{aligned}
$$

where we have used the well known formula

$$
\Gamma(x)=\frac{\pi}{\sin (\pi x) \Gamma(1-x)} .
$$

The string BCJ relation for tachyon derived above is valid for all energies.

For the $N$-point open string tachyon amplitudes $B_{N}$ of Koba-Nielson, some authors in the early days of dual models, see for example [33], discussed symmetry relations among $B_{N}$ functions with different cyclic order external momenta by using monodromy of countour integration of the amplitudes. However, no discussion was addressed for string amplitudes with infinite number of higher spin massive string states, on which we will discuss next.

Since there is no reliable saddle point to calculate $s-t$ channel HSS amplitudes, for all other higher spin string states at arbitrary mass levels, one first calculates the $s-t$ 
channel scattering amplitude with $V_{2}=\alpha_{-1}^{\mu_{1}} \alpha_{-1}^{\mu_{2}} \ldots \alpha_{-1}^{\mu_{n}} \mid 0, k>$, the highest spin state at mass level $M_{2}^{2}=2(N-1)$, and three tachyons $V_{1,3,4}$ as $[8,19]$

$$
\mathcal{T}_{N ; s t}^{\mu_{1} \mu_{2} \cdot \mu_{n}}=\sum_{l=0}^{N}(-)^{l}\left(\begin{array}{c}
N \\
l
\end{array}\right) B\left(-\frac{s}{2}-1+l,-\frac{t}{2}-1+N-l\right) k_{1}^{\left(\mu_{1}\right.} \ldots k_{1}^{\mu_{n-l}} k_{3}^{\mu_{n-l+1}} \ldots k_{3}^{\left.\mu_{N}\right)} .
$$

The corresponding $t-u$ channel open string scattering amplitude can be calculated to be [8]

$$
\mathcal{T}_{N ; t u}^{\mu_{1} \mu_{2} \cdot \mu_{n}}=\sum_{l=0}^{N}\left(\begin{array}{c}
N \\
l
\end{array}\right) B\left(-\frac{t}{2}+N-l-1,-\frac{u}{2}-1\right) k_{1}^{\left(\mu_{1}\right.} \ldots k_{1}^{\mu_{N-l}} k_{3}^{\mu_{N-l+1}} k_{3}^{\left.\mu_{N}\right)} .
$$

The HSS limit of the string BCJ relation for these amplitudes was worked out to be [8]

$$
\mathcal{T}_{N}(s, t) \simeq(-)^{N} \frac{\sin (\pi u / 2)}{\sin (\pi s / 2)} \mathcal{T}_{N}(t, u)
$$

where

$$
\begin{aligned}
\mathcal{T}_{N}(t, u) \simeq & \sqrt{\pi}(-1)^{N-1} 2^{-N} E^{-1-2 N}\left(\sin \frac{\phi}{2}\right)^{-3}\left(\cos \frac{\phi}{2}\right)^{5-2 N} \\
& \cdot \exp \left[-\frac{t \ln t+u \ln u-(t+u) \ln (t+u)}{2}\right]
\end{aligned}
$$

Note that unlike the case of tachyon, this relation was proved only for HSS limit. The next key step was that the result of eq. (2.6) can be generalized to the case of three tachyons and one arbitrary string states $[18,19]$, and then to the case of four arbitrary string states. This generalization was based on the important result that, at each fixed mass level $N$, the high energy fixed angle string scattering amplitudes for states differ from leading Regge trajectory higher spin state in the second vertex are all proportional to each other $[18,19]$

$$
\frac{T_{s t}^{(N, 2 m, q)}}{T_{s t}^{(N, 0,0)}} \simeq \frac{T_{t u}^{(N, 2 m, q)}}{T_{t u}^{(N, 0,0)}}=\left(-\frac{1}{M}\right)^{2 m+q}\left(\frac{1}{2}\right)^{m+q}(2 m-1) ! !
$$

Here $\mathcal{T}_{N}(t, u)=T_{t u}^{(N, 0,0)}$ for the case of three tachyons and one tensor, and $T^{(N, 2 m, q)}$ represents leading order hard open string scattering amplitudes with three arbitrary string states and one higher spin string state of the following form [18, 19]

$$
|N, 2 m, q\rangle \equiv\left(\alpha_{-1}^{T}\right)^{N-2 m-2 q}\left(\alpha_{-1}^{L}\right)^{2 m}\left(\alpha_{-2}^{L}\right)^{q}|0, k\rangle
$$

where the polarizations of the higher spin string state with momentum $k_{2}$ on the scattering plane were defined to be $e^{P}=\frac{1}{M_{2}}\left(E_{2}, \mathrm{k}_{2}, 0\right)=\frac{k_{2}}{M_{2}}, e^{L}=\frac{1}{M_{2}}\left(\mathrm{k}_{2}, E_{2}, 0\right)$ and $e^{T}=(0,0,1)$, and we have omitted possible tensor indices of the other three string states. This high energy symmetry of string theory was first conjectured by Gross in 1988 [11, 12] and was explicitly proved in [14-16, 18-20].

Finally, the string BCJ relations for arbitrary string states in the hard scattering limit can be written as $[8]$

$$
T_{s t}^{(N, 2 m, q)} \simeq(-)^{N} \frac{\sin (\pi u / 2)}{\sin (\pi s / 2)} T_{t u}^{(N, 2 m, q)}=\frac{\sin \left(\pi k_{2} \cdot k_{4}\right)}{\sin \left(\pi k_{1} \cdot k_{2}\right)} T_{t u}^{(N, 2 m, q)}
$$


Eq. (2.8) and eq. (2.10) can be combined to form the extended linear relations in the HSS limit. These relations relate string scattering amplitudes of string states with different spins and different channels in the HSS limit, and can be used to reduce the infinite number of independent hard string scattering amplitudes from $\infty$ down to 1 .

Note that historically the motivation to probe string theory BCJ relations in this context was the calculation of high energy closed string scattering amplitudes [8] by using the KLT relations [22]. Indeed, it was found that the saddle point calculations of high energy fixed angle open string scattering amplitudes were available only for the $t-u$ channel, but not reliable for the $s-t$ channel neither for the closed string high energy amplitude calculation [8]. So it was important to use other method to express $s-t$ channel hard string scattering amplitudes in terms of $t-u$ channel HSS amplitudes.

The factor in eq. (2.10) $\frac{\sin (\pi u / 2)}{\sin (\pi s / 2)}$ which was missing in the literature $[9,10,24]$ has important physical interpretations [8]. The presence of poles give infinite number of resonances in the string spectrum and zeros give the coherence of string scatterings. These poles and zeros survive in the high energy limit and can not be dropped out. Presumably, the factor in the string BCJ relation in eq. (2.10) triggers the failure of saddle point calculation in the $s-t$ channel.

The two relations in eq. (2.2) and eq. (2.10) can be written as the four point string $B C J$ relation which are valid to all energies as

$$
A_{s t}=\frac{\sin \left(\pi k_{2} \cdot k_{4}\right)}{\sin \left(\pi k_{1} k_{2}\right)} A_{t u}
$$

if one can generalize the proof of eq. (2.10) to all energies. This was done in a paper based on monodromy of integration in string amplitude calculation published in 2009 [4]. However, the explicit forms of the amplitudes $A_{s t}$ and $A_{t u}$ were not calculated in [4] and the extended linear relations were not addressed there. In the next section, we will provide an alternative proof of this string BCJ relation.

\section{$2.2 \quad$ Regge string scatterings}

Another interesting regime of the string BCJ relation in eq. (2.11) was the Regge regime. The $s-t$ channel RSS amplitude of three tachyons and one higher spin state in eq. (2.9) was calculated to be [27]

$$
\begin{aligned}
R_{s t}^{(n, 2 m, q)}= & B\left(-1-\frac{s}{2},-1-\frac{t}{2}\right) \sqrt{-t}^{n-2 m-2 q}\left(\frac{1}{2 M_{2}}\right)^{2 m+q} \\
& \cdot 2^{2 m}\left(\tilde{t}^{\prime}\right)^{q} U\left(-2 m, \frac{t}{2}+2-2 m, \frac{\tilde{t}^{\prime}}{2}\right)
\end{aligned}
$$

where $\tilde{t}^{\prime}=t+M_{2}^{2}-M_{3}^{2}$ and $U$ is the Kummer function of the second kind. The corrsponding $t-u$ channel amplitude was calculated to be [23]

$$
\begin{aligned}
R_{t u}^{(n, 2 m, q)}= & (-)^{n} B\left(-1-\frac{t}{2},-1-\frac{u}{2}\right)(\sqrt{-t})^{n-2 m-2 q}\left(\frac{1}{2 M_{2}}\right)^{2 m+q} \\
& \cdot 2^{2 m}\left(\tilde{t}^{\prime}\right)^{q} U\left(-2 m, \frac{t}{2}+2-2 m, \frac{\tilde{t}^{\prime}}{2}\right) .
\end{aligned}
$$


We can calculate the ratio of the two amplitudes as following

$$
\begin{aligned}
\frac{R_{s t}^{(n, 2 m, q)}}{R_{t u}^{(n, 2 m, q)}} & =(-1)^{n} \frac{B\left(-\frac{s}{2}-1,-\frac{t}{2}-1\right)}{B\left(-\frac{t}{2}-1,-\frac{u}{2}-1\right)} \\
& =\frac{(-1)^{n} \Gamma\left(-\frac{s}{2}-1\right) \Gamma\left(\frac{s}{2}+2-n\right)}{\Gamma\left(-\frac{u}{2}-1\right) \Gamma\left(\frac{u}{2}+2-n\right)} \\
& =\frac{(-1)^{n} \Gamma\left(-\frac{s}{2}-1\right) \Gamma\left(\frac{s}{2}+2\right)}{\Gamma\left(-\frac{u}{2}-1\right) \Gamma\left(\frac{u}{2}+2\right)} \cdot \frac{\left(\frac{u}{2}+2-n\right) \cdots\left(\frac{u}{2}+1\right)}{\left(\frac{s}{2}+2-n\right) \cdots\left(\frac{s}{2}+1\right)}
\end{aligned}
$$

One can now take the Regge limit, $t=$ fixed and $s \sim-u \rightarrow \infty$ to get

$$
\frac{R_{s t}^{(n, 2 m, q)}}{R_{t u}^{(n, 2 m, q)}} \simeq \frac{(-1)^{n} \Gamma\left(-\frac{s}{2}-1\right) \Gamma\left(\frac{s}{2}+2\right)}{\Gamma\left(-\frac{u}{2}-1\right) \Gamma\left(\frac{u}{2}+2\right)} \cdot(-1)^{n}=\frac{\sin \pi\left(\frac{u}{2}+2\right)}{\sin \pi\left(\frac{s}{2}+2\right)}=\frac{\sin \pi\left(k_{2} \cdot k_{4}\right)}{\sin \pi\left(k_{1} \cdot k_{2}\right)},
$$

which can be written as

$$
R_{s t}^{(n, 2 m, q)} \simeq \frac{\sin \left(\pi k_{2} \cdot k_{4}\right)}{\sin \left(\pi k_{1} \cdot k_{2}\right)} R^{(n, 2 m, q)}(t, u),
$$

and is consistent with the string BCJ relation in eq. (2.11).

The result in eq. (2.16) can be generalized to the general leading order Regge string states at each fixed mass level $N=\sum_{n, m, l>0} n p_{n}+m q_{m}+l r_{l}[27]$

$$
\left|p_{n}, q_{m}, r_{l}\right\rangle=\prod_{n>0}\left(\alpha_{-n}^{T}\right)^{p_{n}} \prod_{m>0}\left(\alpha_{-m}^{P}\right)^{q_{m}} \prod_{l>0}\left(\alpha_{-l}^{L}\right)^{r_{l}}|0, k\rangle .
$$

For this general case, the $s-t$ channel of the scattering amplitude in the Regge limit was calculated to be $[26]$

$$
\begin{aligned}
R_{s t}^{\left|p_{n}, q_{m}, r_{l}\right\rangle}= & \prod_{n=1}[(n-1) ! \sqrt{-t}]^{p_{n}} \prod_{m=1}\left[(m-1) ! \frac{\tilde{t}}{2 M_{2}}\right]^{q_{m}} \prod_{l=1}\left[(l-1) ! \frac{\tilde{t}^{\prime}}{2 M_{2}}\right]^{r_{l}} \\
& \cdot F_{1}\left(-\frac{t}{2}-1 ;-q_{1},-r_{1} ; \frac{s}{2} ;-\frac{s}{\tilde{t}},-\frac{s}{\tilde{t}^{\prime}}\right) B\left(-\frac{s}{2}-1,-\frac{t}{2}-1\right)
\end{aligned}
$$

where $F_{1}$ is the first Appell function. On the other hand, one can calculate the $t-u$ channel amplitude, which was missing in [26], as following

$$
\begin{aligned}
R_{t u}^{\left|p_{n}, q_{m}, r_{l}\right\rangle}= & \int_{1}^{\infty} d x \cdot x^{k_{1} \cdot k_{2}}(x-1)^{k_{2} \cdot k_{3}}\left[\frac{k_{1}^{P}}{x}-\frac{k_{3}^{P}}{1-x}\right]^{q_{1}}\left[\frac{k_{1}^{L}}{x}-\frac{k_{3}^{L}}{1-x}\right]^{r_{l}} \\
& \cdot \prod_{n=1}\left[-\frac{(n-1) ! k_{3}^{T}}{(1-x)^{n}}\right] \prod_{m=2}\left[-\frac{(m-1) ! k_{3}^{P}}{(1-x)^{m}}\right] \prod_{l=2}\left[-\frac{(l-1) ! k_{3}^{L}}{(1-x)^{l}}\right] \\
= & \prod_{n=1}[(n-1) ! \sqrt{-t}]^{p_{n}} \prod_{m=1}\left[(m-1) ! \frac{\tilde{t}}{2 M_{2}}\right]^{q_{m}} \prod_{l=1}\left[(l-1) ! \frac{\tilde{t}^{\prime}}{2 M_{2}}\right]^{r_{l}} \\
& \cdot \sum_{i}^{q_{1}}\left(\begin{array}{c}
q_{1} \\
i
\end{array}\right)\left(-\frac{s}{\tilde{t}}\right)^{i} \sum_{j}^{r_{1}}\left(\begin{array}{c}
r_{1} \\
j
\end{array}\right)\left(-\frac{s}{\tilde{t}^{\prime}}\right)^{j}(-1)^{k_{2} \cdot k_{3}}
\end{aligned}
$$




$$
\cdot \int_{1}^{\infty} d x \cdot x^{-\frac{s}{2}-2+N-i-j}(1-x)^{-\frac{t}{2}-2+i+j}
$$

We can do the change of variable $y=\frac{x-1}{x}$ to get

$$
\begin{aligned}
R_{t u}^{\left|p_{n}, q_{m}, r_{l}\right\rangle}= & (-1)^{N} \prod_{n=1}[(n-1) ! \sqrt{-t}]^{p_{n}} \prod_{m=1}\left[(m-1) ! \frac{\tilde{t}}{2 M_{2}}\right]^{q_{m}} \prod_{l=1}\left[(l-1) ! \frac{\tilde{t}^{\prime}}{2 M_{2}}\right]^{r_{l}} \\
& \cdot \sum_{i}^{q_{1}}\left(\begin{array}{c}
q_{1} \\
i
\end{array}\right)\left(-\frac{s}{\tilde{t}}\right)^{i} \sum_{j}^{r_{1}}\left(\begin{array}{c}
r_{1} \\
j
\end{array}\right)\left(-\frac{s}{\tilde{t}^{\prime}}\right)^{j}(-1)^{-i-j} \int_{0}^{1} d y \cdot y^{\frac{-t}{2}-2+i+j}(1-y)^{\frac{-u}{2}-2} \\
= & (-1)^{N} \prod_{n=1}[(n-1) ! \sqrt{-t}]^{p_{n}} \prod_{m=1}\left[(m-1) ! \frac{\tilde{t}}{2 M_{2}}\right]^{q_{m}} \prod_{l=1}\left[(l-1) ! \frac{\tilde{t}^{\prime}}{2 M_{2}}\right]^{r_{l}} \\
& \cdot \sum_{i}^{q_{1}}\left(\begin{array}{c}
q_{1} \\
i
\end{array}\right)\left(\frac{-s}{\tilde{t}}\right)^{i} \sum_{j}^{r_{1}}\left(\begin{array}{c}
r_{1} \\
j
\end{array}\right)\left(\frac{-s}{\tilde{t}^{\prime}}\right)^{j}(-1)^{-i-j} B\left(\frac{-t}{2}-1+i+j, \frac{-u}{2}-1\right)
\end{aligned}
$$

In the Regge limit, $t=$ fixed and $s \sim-u \rightarrow \infty$, one gets

$$
\begin{aligned}
R_{t u}^{\left|p_{n}, q_{m}, r_{l}\right\rangle} \simeq & (-1)^{N} \prod_{n=1}[(n-1) ! \sqrt{-t}]^{p_{n}} \prod_{m=1}\left[(m-1) ! \frac{\tilde{t}}{2 M_{2}}\right]^{q_{m}} \prod_{l=1}\left[(l-1) ! \frac{\tilde{t}^{\prime}}{2 M_{2}}\right]^{r_{l}} \\
& \cdot F_{1}\left(-\frac{t}{2}-1 ;-q_{1},-r_{1} ; \frac{s}{2} ;-\frac{s}{\tilde{t}},-\frac{s}{\tilde{t}^{\prime}}\right) B\left(-\frac{t}{2}-1,-\frac{u}{2}-1+N\right) \cdot
\end{aligned}
$$

Finally, one can derive the string BCJ relation in the Regge limit

$$
\frac{R_{s t}^{\left|p_{n}, q_{m}, r_{l}\right\rangle}}{R_{t u}^{\left|p_{n}, q_{m}, r_{l}\right\rangle}}=\frac{(-1)^{N} B\left(-\frac{s}{2}-1,-\frac{t}{2}-1\right)}{B\left(-\frac{t}{2}-1,-\frac{u}{2}-1+N\right)}=\frac{\sin \pi\left(\frac{u}{2}+2-N\right)}{(-1)^{N} \sin \pi\left(\frac{s}{2}+2\right)}=\frac{\sin k_{2} \cdot k_{4}}{\sin k_{1} \cdot k_{2}} .
$$

In constrast to the linear relations calculated in the hard scattering limit in eq. (2.8), it was shown $[25,26]$ that there existed infinite recurrence relation among RSS amplitudes. For example, the recurrence relation $[26]\left(\left(N ; q_{1}, r_{1}\right)\right.$ etc. refer to states in eq. $\left.(2.9)\right)$

$$
\sqrt{-t}\left[R_{s t}^{\left(N ; q_{1}, r_{1}\right)}+R_{s t}^{\left(N ; q_{1}-1, r_{1}+1\right)}\right]-M R_{s t}^{\left(N ; q_{1}-1, r_{1}\right)}=0
$$

for arbitrary mass levels $M^{2}=2(N-1)$ can be derived from recurrence relations of the Appell functions. Eq. (2.22) and eq. (2.23) can be combined to form one example of the extended recurrence relations in the RSS limit. The possible connection of field theory BCJ relations [3] and Regge string recurrence relations [25] was first suggested in [25]. These relations relate string scattering amplitudes of string states with different spins and different channels in the RSS limit. Similar to the HSS limit, it can be shown $[25,26]$ that the complete extended recurrence relations in the RSS limit can be used to reduce the infinite number of independent RSS amplitudes from $\infty$ down to 1.

We have seen in this section that the HSS and the RSS amplitudes calculated previously are consistent with the string BCJ relation in eq. (2.11). In the next section, we will give an explicit proof the string BCJ relation for all energies. In addition in section IV, we will calculate the nonrelativistic limit of string BCJ relation to obtain the extended recurrence relation in the nonrelativistic scattering limit. 


\section{Explicit proof of string BCJ}

In this section, we generalize the explicit calculations of high energy four point string BCJ relations reviewed in the last section to all energy. The proof of $n$-point string BCJ relations using monodromy was given in [4] without calculating string amplitudes, Here we will explicitly calculate string scattering amplitudes for four arbitrary string states for both $s-t$ and $t-u$ channels and directly prove the four point string BCJ relations.

There are at least two motivations to calculate the string BCJ relation explicitly and give an alternative proof of the relations. Firstly, the proof in [4] assumed negative real parts of $k_{i} \cdot k_{j}$, and puts some constraints on the kinematic regime for the validity of the string BCJ relations. Our explicit proof here is on the contrary valid for all kinematic regimes. Secondly, in section II the explicit calculations of scattering amplitudes in the high energy string BCJ relation had led to the extended relations both in the hard scattering limit and the Regge limit. As we will see in section IV, the explicit calculation of the string BCJ relation in the nonrelativistic scattering limit will also lead to new recurrence relations among low energy string scattering amplitudes. Similarly, we will see along the calculation of this section that the equality of the string BCJ relations can be identified as the equalities of coefficients of two multi-linear polynomials of $k_{1}^{\mu}$ and $k_{3}^{\nu}$ in the $s-t$ and $t-u$ channel amplitudes.

Instead of path integral approach, we will use the method of Wick contraction to do the open string scattering amplitude calculation. As usual, we will be fixing $\operatorname{SL}(2, R)$ by choosing string worldsheet coordinates to be $x_{1}=0, x_{3}=1, x_{4}=\infty$. We first give the answer of a simple example $\left(\alpha^{\prime}=\frac{1}{2} ; s-t\right.$ channel $)$

$$
\begin{aligned}
\mathcal{T}^{\mu \nu}= & \int \prod_{i=1}^{4} d x_{i}<e^{i k_{1} X} \partial^{2} X^{(\mu} \partial X^{\nu)} e^{i k_{2} X} e^{i k_{3} X} e^{i k_{4} X}> \\
= & \frac{\Gamma\left(-\frac{s}{2}-1\right) \Gamma\left(-\frac{t}{2}-1\right)}{\Gamma\left(\frac{u}{2}+2\right)}\left[\frac{t}{2}\left(\frac{t^{2}}{4}-1\right) k_{1}^{\mu} k_{1}^{\nu}-\left(\frac{s}{2}+1\right) \frac{t}{2}\left(\frac{t}{2}+1\right) k_{1}^{\mu} k_{3}^{\nu}\right. \\
& \left.\quad+\frac{s}{2}\left(\frac{s}{2}+1\right)\left(\frac{t}{2}+1\right) k_{3}^{\mu} k_{1}^{\nu}-\frac{s}{2}\left(\frac{s^{2}}{4}-1\right) k_{3}^{\mu} k_{3}^{\nu}\right] .
\end{aligned}
$$

The result is a multi-linear polynomial of $k_{1}^{\mu}$ and $k_{3}^{\nu}$ due to the choice of worldsheet coordinates above. To prove the equality of $s-t$ and $t-u$ channel calculation, we can just show the equality of coefficients of a typical term in each channel.

There are two key observations before we proceed to do the calculation. Firstly, we can drop out the fourth vertex $V_{4}\left(x_{4}\right)$ in the real calculation due to the choice $x_{4}=\infty$. Secondly, there are two types of contributions in the contractions between two vertex operators. They are contraction between $\partial^{a} X$ and $\partial^{a^{\prime}} X$, and contraction between $\partial^{a} X$ and $e^{i k X}$.

we are going to calculate the most general four point function of string vertex

$$
\begin{aligned}
& \left\langle V_{1}\left(x_{1}\right) V_{2}\left(x_{2}\right) V_{3}\left(x_{3}\right) V_{4}\left(x_{4}\right)\right\rangle
\end{aligned}
$$

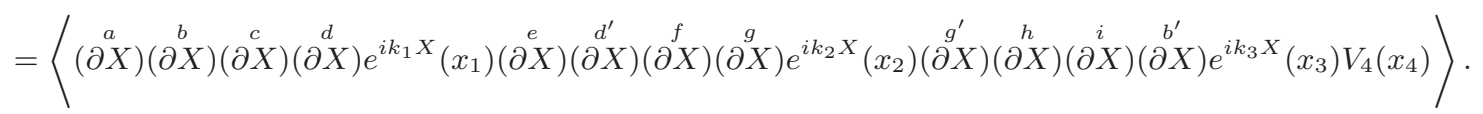


We can write down the relavent three vertex operators as

$$
\begin{aligned}
& V_{1}\left(x_{1}\right)=(\stackrel{a}{\partial} X)(\stackrel{b}{\partial} X)(\stackrel{c}{\partial} X)(\stackrel{d}{\partial X}) e^{i k_{1} X}\left(x_{1}\right), \\
& V_{2}\left(x_{2}\right)=(\stackrel{e}{\partial X})\left(\stackrel{d^{\prime}}{\partial X} \stackrel{f}{f}^{\prime}(\stackrel{g}{\partial})(\stackrel{g}{\partial X}) e^{i k_{2} X}\left(x_{2}\right),\right. \\
& V_{3}\left(x_{3}\right)=\left(\stackrel{g^{\prime}}{\partial X}\right)(\stackrel{h}{\partial} X)\left(\partial \stackrel{i}{b^{\prime}}\right)(\partial X) e^{i k_{3} X}\left(x_{3}\right),
\end{aligned}
$$

where

$$
\begin{aligned}
& (\partial \dot{a} X)=\prod_{a=1}^{A}\left(i \varepsilon_{11 a}^{(a)} \cdot \partial_{1}^{\alpha_{11 a}} X_{1}\right), \quad\left(\partial{ }^{b} X\right)=\prod_{b=1}^{B}\left(i \varepsilon_{12 b}^{(b)} \cdot \partial_{1}^{\alpha_{12 b}} X_{1}\right), \quad(\partial X)=\prod_{c=1}^{C}\left(i \varepsilon_{13 c}^{(c)} \cdot \partial_{1}^{\alpha_{13 c}} X_{1}\right), \\
& (\partial \stackrel{d}{X})=\prod_{d=1}^{D}\left(i \varepsilon_{14 d}^{(d)} \cdot \partial_{1}^{\alpha_{14 d}} X_{1}\right), \quad(\partial X)=\prod_{e=1}^{E}\left(i \varepsilon_{21 a}^{(e)} \cdot \partial_{2}^{\alpha_{21 e}} X_{2}\right), \quad\left(\stackrel{d^{\prime \prime}}{\partial X}\right)=\prod_{d^{\prime}=1}^{D}\left(i \varepsilon_{22 b}^{\left(d^{\prime}\right)} \cdot \partial_{2}^{\alpha_{22 d^{\prime}}} X_{2}\right), \\
& (\stackrel{f}{\partial X})=\prod_{f=1}^{F}\left(i \varepsilon_{23 f}^{(f)} \cdot \partial_{2}^{\alpha_{23 f}} X_{2}\right), \quad(\partial X)=\prod_{g=1}^{G}\left(i \varepsilon_{24 g}^{(g)} \cdot \partial_{2}^{\alpha_{24 g}} X_{2}\right), \quad\left(\stackrel{g^{\prime}}{\partial X}\right)=\prod_{g^{\prime}=1}^{G}\left(i \varepsilon_{31 e}^{\left(e^{\prime}\right)} \cdot \partial_{3}^{\alpha_{31 g^{\prime}}} X_{3}\right), \\
& (\partial \stackrel{h}{h})=\prod_{h=1}^{H}\left(i \varepsilon_{32 h}^{(h)} \cdot \partial_{3}^{\alpha_{32 h}} X_{3}\right), \quad(\partial X)=\prod_{i=1}^{I}\left(i \varepsilon_{33 i}^{(i)} \cdot \partial_{3}^{\alpha_{33 i}} X_{3}\right), \quad\left(\partial^{b^{\prime}}\right)=\prod_{b^{\prime}=1}^{B}\left(i \varepsilon_{34 b}^{\left(b^{\prime}\right)} \cdot \partial_{3}^{\alpha_{34 b}} X_{3}\right) .
\end{aligned}
$$

In eq. (3.4), $\varepsilon_{11 a}^{(a)}$ and $\alpha_{11 a}$ etc. are polarizations and orders of worldsheet differential respectively. In eq. (3.3), we have four groups of $\partial^{a} X$ for each vertex operator. Two of them will contract with $\partial^{b} X$ of the other two vertex operators respectively, and the rest two will contract with $e^{i k X}$ of the other two vertex operators respectively. For illustration, we have used the pair dummy indexes $b, b^{\prime} ; d, d^{\prime}$ and $g, g^{\prime}$ for contractions. The other six indexes $a, c, e, f, h$ and $i$ are prepared for contractions with $e^{i k X}$.

The mass levels of the three vertex operators are

$$
\begin{aligned}
& N_{1}=S_{A}+S_{B}+S_{C}+S_{D}, \\
& N_{2}=S_{E}+S_{D}^{\prime}+S_{F}+S_{G}, \\
& N_{3}=S_{G}^{\prime}+S_{H}+S_{I}+S_{B}^{\prime},
\end{aligned}
$$

where we defined

$$
\begin{aligned}
S_{A}=\sum_{a=1}^{A} \alpha_{11 a}, & S_{B}=\sum_{b=1}^{B} \alpha_{12 b}, & S_{C}=\sum_{c=1}^{C} \alpha_{13 c}, & S_{D}=\sum_{d=1}^{D} \alpha_{14 d}, \\
S_{E}=\sum_{e=1}^{E} \alpha_{21 e}, & S_{F}=\sum_{f=1}^{F} \alpha_{23 f}, & S_{G}=\sum_{g=1}^{G} \alpha_{24 g}, & S_{H}=\sum_{h=1}^{H} \alpha_{32 h}, \\
S_{I}=\sum_{i=1}^{I} \alpha_{33 i}, & S_{B}^{\prime}=\sum_{b^{\prime}=1}^{B} \alpha_{34 b^{\prime}}, & S_{D}^{\prime}=\sum_{d^{\prime}=1}^{D} \alpha_{22 d^{\prime}}, & S_{G}^{\prime}=\sum_{g^{\prime}=1}^{G} \alpha_{31 g^{\prime}} .
\end{aligned}
$$

Then we have

$$
\begin{aligned}
s & =-\left(k_{1}+k_{2}\right)^{2}, \quad t=-\left(k_{2}+k_{3}\right)^{2}, \quad u=-\left(k_{1}+k_{3}\right)^{2}, \\
k_{1} \cdot k_{2} & =-\frac{s}{2}-2+N_{1}+N_{2}
\end{aligned}
$$




$$
\begin{aligned}
k_{2} \cdot k_{3} & =-\frac{t}{2}-2+N_{2}+N_{3}, \\
k_{1} \cdot k_{3} & =-\frac{u}{2}-2+N_{1}+N_{3}, \\
s+t+u & =2 N^{\prime}-8, \quad \text { with } N^{\prime}=N_{1}+N_{2}+N_{3}+N_{4} .
\end{aligned}
$$

We are now ready to do the calculation. After putting the $\operatorname{SL}(2, R)$ gauge choice, we get

$$
\begin{aligned}
T= & \prod_{a=1}^{A}\left[\varepsilon_{11 a}^{(a)} \cdot k_{3}(-1)\left(\alpha_{11 a}-1\right) !\right] \prod_{b=1}^{B}\left[\varepsilon_{12 b}^{(b)} \cdot \varepsilon_{34 b}^{(b)}(-1)^{\alpha_{34 b}-1}\left(\alpha_{12 b}+\alpha_{34 b}-1\right) !\right] \\
& \cdot \prod_{c=1}^{C}\left[\varepsilon_{13 c}^{(c)} \cdot k_{2}(-1)\left(\alpha_{13 c}-1\right) !\right] \prod_{d=1}^{D}\left[\varepsilon_{14 d}^{(d)} \cdot \varepsilon_{22 d}^{(d)}(-1)^{\alpha_{22 d}-1}\left(\alpha_{14 d}+\alpha_{22 d}-1\right) !\right] \\
& \cdot \prod_{e=1}^{E}\left[\varepsilon_{21 e}^{(e)} \cdot k_{1}(-1)^{\alpha_{21 e}}\left(\alpha_{21 e}-1\right) !\right] \prod_{f=1}^{F}\left[\varepsilon_{23 f}^{(f)} \cdot k_{3}(-1)\left(\alpha_{23 f}-1\right) !\right] \\
& \cdot \prod_{g=1}^{G}\left[\varepsilon_{24 g}^{(g)} \cdot \varepsilon_{31 g}^{(g)}(-1)^{\alpha_{31 g}-1}\left(\alpha_{24 g}+\alpha_{31 g}-1\right) !\right] \prod_{h=1}^{H}\left[\varepsilon_{32 h}^{(h)} \cdot k_{2}(-1)\left(\alpha_{32 h}-1\right) !\right] \\
& \cdot \prod_{i=1}^{I}\left[\varepsilon_{33 i}^{(i)} \cdot k_{1}(-1)^{\alpha_{33 i}}\left(\alpha_{33 i}-1\right) !\right] \\
& \cdot \int d x|-x|^{k_{1} \cdot k_{2}}|x-1|^{k_{2} \cdot k_{3}} x^{-\left(S_{C}+S_{D}+S_{D}^{\prime}+S_{E}\right)}(1-x)^{-\left(S_{F}+S_{G}+S_{G}^{\prime}+S_{H}\right)} .
\end{aligned}
$$

It is easy to see that only the last term of eq. (3.8) will be different for the $s-t$ and $t-u$ channel calculations. For the $s-t$ channel, the last term becomes

$$
\begin{array}{rl}
\int_{0}^{1} d & x x^{k_{1} \cdot k_{2}}(1-x)^{k_{2} \cdot k_{3}} x^{-\left(S_{C}+S_{D}+S_{D}^{\prime}+S_{E}\right)}(1-x)^{-\left(S_{F}+S_{G}+S_{G}^{\prime}+S_{H}\right)} \\
& =\int_{0}^{1} d x \times x^{-\frac{s}{2}-2+S_{A}+S_{B}+S_{F}+S_{G}}(1-x)^{-\frac{t}{2}-2+S_{E}+S_{D}+S_{I}+S_{B}^{\prime}} \\
& =\frac{\Gamma\left(\frac{-s}{2}-1+S_{A}+S_{B}+S_{F}+S_{G}\right) \Gamma\left(-\frac{t}{2}-1+S_{E}+S_{D}^{\prime}+S_{I}+S_{B}^{\prime}\right)}{\Gamma\left(\frac{u}{2}+2-N_{4}-S_{C}-S_{D}-S_{G}^{\prime}-S_{H}\right)} .
\end{array}
$$

For the $t-u$ channel, we have the last term

$$
\int_{1}^{\infty} d x(x)^{k_{1} \cdot k_{2}}(x-1)^{k_{2} \cdot k_{3}} x^{-\left(S_{C}+S_{D}+S_{D}^{\prime}+S_{E}\right)}(1-x)^{-\left(S_{F}+S_{G}+S_{G}^{\prime}+S_{H}\right)} .
$$

Define

$$
K=-\left(S_{F}+S_{G}+S_{G}^{\prime}+S_{H}\right)
$$

and make the chane of variable $x=\frac{1}{1-y}$ in the integration, we end up with

$$
\begin{aligned}
& (-1)^{K} \int_{0}^{1} d y(y)^{\frac{-t}{2}-2+S_{E}+S_{D}+S_{I}+S_{B}^{\prime}}(1-y)^{-\frac{u}{2}-2+N_{4}+S_{C}+S_{D}+S_{G}^{\prime}+S_{H}} \\
& =(-1)^{K} \frac{\Gamma\left(\frac{-t}{2}-1+S_{E}+S_{D}+S_{I}+S_{B}^{\prime}\right) \Gamma\left(-\frac{u}{2}-1+N_{4}+S_{C}+S_{D}+S_{G}^{\prime}+S_{H}\right)}{\Gamma\left(\frac{s}{2}+2-S_{A}-S_{B}-S_{F}-S_{G}\right)}
\end{aligned}
$$


We are now ready to calculate the ratio

$$
\begin{aligned}
& \frac{T_{s t}}{T_{t u}}=\frac{(-1)^{K} \Gamma\left(\frac{-s}{2}-1+S_{A}+S_{B}+S_{F}+S_{G}\right) \Gamma\left(\frac{s}{2}+2-S_{A}-S_{B}-S_{F}-S_{G}\right)}{\Gamma\left(-\frac{u}{2}-1+N_{4}+S_{C}+S_{D}+S_{G}^{\prime}+S_{H}\right) \Gamma\left(\frac{u}{2}+2-N_{4}-S_{C}-S_{D}-S_{G}^{\prime}-S_{H}\right)} \\
& =(-1)^{-\left(S_{F}+S_{G}+S_{G}^{\prime}+S_{H}\right)} \cdot \frac{\sin \pi\left(\frac{u}{2}+2-N_{4}-S_{C}-S_{D}-S_{G}^{\prime}-S_{H}\right)}{\sin \pi\left(\frac{s}{2}+2-S_{A}-S_{B}-S_{F}-S_{G}\right)} \\
& =(-1)^{-N_{1}-N_{4}} \frac{\sin \pi\left(\frac{u}{2}+2\right)}{\sin \pi\left(\frac{s}{2}+2\right)}=\frac{\sin \pi\left(k_{2} \cdot k_{4}\right)}{\sin \pi\left(k_{1} \cdot k_{2}\right)},
\end{aligned}
$$

where we have used the identity (2.3). We thus have provd the four point string BCJ relation by explicit calculation.

\section{Nonrelativistic string BCJ and extended recurrence relations}

In this section, in constrast to the two high energy limits of string BCJ relations discussed in section II, we discuss mass level dependent nonrelativistic string BCJ relations. For simplicity, we will first calculate both $s-t$ and $t-u$ channel NSS amplitudes of three tachyons and one leading trojectory string state at arbitrary mass levels. We will then calculate NSS amplitudes of three tachyons and one more general string state. We will see that the mass and spin dependent nonrelativistic string BCJ relations can be expressed in terms of Gauss hypergeometry functions. As an application, for each fixed mass level $N$ we can then derive extended recurrence relations among NSS amplitudes of string states with different spins and different channels.

We choose $k_{2}$ to be momentum of the leading trojectory string states and the rest are tachyons. In the CM frame

$$
\begin{aligned}
& k_{1}=\left(\sqrt{M_{1}^{2}+{\overrightarrow{k_{1}}}^{2}},-\left|\overrightarrow{k_{1}}\right|, 0\right), \\
& k_{2}=\left(\sqrt{M_{2}^{2}+{\overrightarrow{k_{1}}}^{2}},+\left|\overrightarrow{k_{1}}\right|, 0\right), \\
& k_{3}=\left(\sqrt{M_{3}^{2}+{\overrightarrow{k_{3}}}^{2}},-\left|\overrightarrow{k_{3}}\right| \cos \phi,-\left|\overrightarrow{k_{3}}\right| \sin \phi\right), \\
& k_{4}=\left(\sqrt{M_{3}^{2}+{\overrightarrow{k_{3}}}^{2}},+\left|\overrightarrow{k_{3}}\right| \cos \phi,+\left|\overrightarrow{k_{3}}\right| \sin \phi\right)
\end{aligned}
$$

where $M_{1}=M_{3}=M_{4}=M_{\text {tachyon }}, M_{2}=2(N-1)$ and $\phi$ is the scattering angle on the scattering plane. Instead of the zero slope limt which was used in the literature to get the field theory limit of the lowest mass string state [28-32], we will take the nonrelativistic $\left|\overrightarrow{k_{1}}\right| \ll M_{S}$ or large $M_{S}$ limit for the massive string scattering amplitudes. In the nonrelativistic limit $\left(\left|\overrightarrow{k_{1}}\right| \ll M_{S}\right)$

$$
\begin{aligned}
& k_{1} \simeq\left(M_{1}+\frac{{\overrightarrow{k_{1}}}^{2}}{2 M_{1}},-\left|\overrightarrow{k_{1}}\right|, 0\right), \\
& k_{2} \simeq\left(M_{2}+\frac{{\overrightarrow{k_{1}}}^{2}}{2 M_{2}},+\left|\overrightarrow{k_{1}}\right|, 0\right),
\end{aligned}
$$




$$
\begin{aligned}
k_{3} \simeq & \left(-\frac{M_{1}+M_{2}}{2}-\frac{1}{4} \frac{M_{1}+M_{2}}{M_{1} M_{2}}\left|\overrightarrow{k_{1}}\right|^{2},-\left[\frac{\epsilon}{2}+\frac{\left(M_{1}+M_{2}\right)}{4 M_{1} M_{2} \epsilon}\left|\overrightarrow{k_{1}}\right|^{2}\right] \cos \phi,\right. \\
& \left.-\left[\frac{\epsilon}{2}+\frac{\left(M_{1}+M_{2}\right)}{4 M_{1} M_{2} \epsilon}\left|\overrightarrow{k_{1}}\right|^{2}\right] \sin \phi\right), \\
k_{4} \simeq & \left(-\frac{M_{1}+M_{2}}{2}-\frac{1}{4} \frac{M_{1}+M_{2}}{M_{1} M_{2}}\left|\overrightarrow{k_{1}}\right|^{2},+\left[\frac{\epsilon}{2}+\frac{\left(M_{1}+M_{2}\right)}{4 M_{1} M_{2} \epsilon}\left|\overrightarrow{k_{1}}\right|^{2}\right] \cos \phi,\right. \\
& \left.+\left[\frac{\epsilon}{2}+\frac{\left(M_{1}+M_{2}\right)}{4 M_{1} M_{2} \epsilon}\left|\overrightarrow{k_{1}}\right|^{2}\right] \sin \phi\right) .
\end{aligned}
$$

where

$$
\epsilon=\sqrt{\left(M_{1}+M_{2}\right)^{2}-4 M_{3}^{2}} .
$$

The three polarizations on the scattering plane are defined to be [14-16]

$$
\begin{aligned}
e^{P} & =\frac{1}{M_{2}}\left(\sqrt{M_{2}^{2}+{\overrightarrow{k_{1}}}^{2}},\left|\overrightarrow{k_{1}}\right|, 0\right), \\
e^{L} & =\frac{1}{M_{2}}\left(\left|\overrightarrow{k_{1}}\right|, \sqrt{M_{2}^{2}+{\overrightarrow{k_{1}}}^{2}}, 0\right), \\
e^{T} & =(0,0,1),
\end{aligned}
$$

which in the low energy limit reduce to

$$
\begin{aligned}
e^{P} & \simeq \frac{1}{M_{2}}\left(M_{2}+\frac{{\overrightarrow{k_{1}}}^{2}}{2 M_{2}},\left|\overrightarrow{k_{1}}\right|, 0\right), \\
e^{L} & \simeq \frac{1}{M_{2}}\left(\left|\overrightarrow{k_{1}}\right|, M_{2}+\frac{{\overrightarrow{k_{1}}}^{2}}{2 M_{2}}, 0\right), \\
e^{T} & \simeq(0,0,1) .
\end{aligned}
$$

One can then calculate the following kinematics which will be used in the low energy amplitude calculation

$$
\begin{aligned}
& k_{1} \cdot e^{L}=k_{1}^{L}=\frac{-\left(M_{1}+M_{2}\right)}{M_{2}}\left|\overrightarrow{k_{1}}\right|+O\left(\left|\overrightarrow{k_{1}}\right|^{2}\right), \\
& k_{3} \cdot e^{L}=k_{3}^{L}=\frac{-\epsilon}{2} \cos \phi+\frac{M_{1}+M_{2}}{2 M_{2}}\left|\overrightarrow{k_{1}}\right|+O\left(\left|\overrightarrow{k_{1}}\right|^{2}\right), \\
& k_{1} \cdot e^{T}=k_{1}^{T}=0, \\
& k_{3} \cdot e^{T}=k_{3}^{T}=\frac{-\epsilon}{2} \sin \phi+O\left(\left|\overrightarrow{k_{1}}\right|^{2}\right), \\
& k_{1} \cdot e^{P}=k_{1}^{P}=-M_{1}+O\left(\left|\overrightarrow{k_{1}}\right|^{2}\right), \\
& k_{3} \cdot e^{P}=k_{3}^{P}=\frac{M_{1}+M_{2}}{2}-\frac{\epsilon}{2 M_{2}} \cos \phi\left|\overrightarrow{k_{1}}\right|+O\left(\left|\overrightarrow{k_{1}}\right|^{2}\right),
\end{aligned}
$$

amd the Mandelstam variables

$$
\begin{aligned}
& s=\left(M_{1}+M_{2}\right)^{2}+O\left(\left|\overrightarrow{k_{1}}\right|^{2}\right), \\
& t=-M_{1} M_{2}-2-\epsilon \cos \phi\left|\overrightarrow{k_{1}}\right|+O\left(\left|\overrightarrow{k_{1}}\right|^{2}\right), \\
& u=-M_{1} M_{2}-2+\epsilon \cos \phi\left|\overrightarrow{k_{1}}\right|+O\left(\left|\overrightarrow{k_{1}}\right|^{2}\right) .
\end{aligned}
$$




\subsection{Leading trojectory states}

We first calculate the nonrelativistic $s-t$ channel scattering amplitude of three tachyons and one tensor string state

$$
V_{2}=\left(i \partial X^{T}\right)^{p}\left(i \partial X^{L}\right)^{r}\left(i \partial X^{P}\right)^{q} e^{i k_{2} X} .
$$

where

$$
N=p+r+q .
$$

To the leading order in energy, the nonrelativistic amplitude can be calculated to be,

$$
\begin{aligned}
A_{s t}^{(p, r, q)}= & \int_{0}^{1} d x\left(\frac{k_{1}^{T}}{x}-\frac{k_{3}^{T}}{1-x}\right)^{p}\left(\frac{k_{1}^{L}}{x}-\frac{k_{3}^{L}}{1-x}\right)^{r}\left(\frac{k_{1}^{P}}{x}-\frac{k_{3}^{P}}{1-x}\right)^{q}|x|^{k_{1} \cdot k_{2}}|x-1|^{k_{2} \cdot k_{3}} \\
\simeq & \int_{0}^{1} d x\left(\frac{\frac{\epsilon}{2} \sin \phi}{1-x}\right)^{p}\left(\frac{\frac{\epsilon}{2} \cos \phi}{1-x}\right)^{r}\left(-\frac{M_{1}}{x}-\frac{\frac{M_{1}+M_{2}}{2}}{1-x}\right)^{q} x^{k_{1} \cdot k_{2}}(1-x)^{k_{2} \cdot k_{3}} \\
= & \left(\frac{\epsilon}{2} \sin \phi\right)^{p}\left(\frac{\epsilon}{2} \cos \phi\right)^{r}\left(-\frac{M_{1}+M_{2}}{2}\right)^{q} \cdot \sum_{l=0}^{q}\left(\begin{array}{l}
q \\
l
\end{array}\right)\left(\frac{2 M_{1}}{M_{1}+M_{2}}\right)^{l} \\
& \cdot B\left(1-M_{1} M_{2}-l, \frac{M_{1} M_{2}}{2}+l\right) \\
= & \left(\frac{\epsilon}{2} \sin \phi\right)^{p}\left(\frac{\epsilon}{2} \cos \phi\right)^{r}\left(-\frac{M_{1}+M_{2}}{2}\right)^{q} B\left(1-M_{1} M_{2}, \frac{M_{1} M_{2}}{2}\right) \\
& \cdot \sum_{l=0}^{q}(-1)^{l}\left(\begin{array}{l}
q \\
l
\end{array}\right)\left(\frac{2 M_{1}}{M_{1}+M_{2}}\right)^{l} \frac{\left(\frac{M_{1} M_{2}}{2}\right)_{l}}{\left(M_{1} M_{2}\right)_{l}} .
\end{aligned}
$$

Finally the summation above can be performed to get the Gauss hypergeometry function ${ }_{2} F_{1}$,

$$
\begin{aligned}
A_{s t}^{(p, r, q)}= & \left(\frac{\epsilon}{2} \sin \phi\right)^{p}\left(\frac{\epsilon}{2} \cos \phi\right)^{r}\left(-\frac{M_{1}+M_{2}}{2}\right)^{q} B\left(1-M_{1} M_{2}, \frac{M_{1} M_{2}}{2}\right) \\
& \cdot{ }_{2} F_{1}\left(\frac{M_{1} M_{2}}{2} ;-q ; M_{1} M_{2} ; \frac{2 M_{1}}{M_{1}+M_{2}}\right) .
\end{aligned}
$$

Similarly, we calculate the corresponding nonrelativistic $t-u$ channel amplitude as,

$$
\begin{aligned}
A_{t u}^{(p, r, q)}= & \int_{1}^{\infty} d x\left(\frac{k_{1}^{T}}{x}-\frac{k_{3}^{T}}{1-x}\right)^{p}\left(\frac{k_{1}^{L}}{x}-\frac{k_{3}^{L}}{1-x}\right)^{r}\left(\frac{k_{1}^{P}}{x}-\frac{k_{3}^{P}}{1-x}\right)^{q}|x|^{k_{1} \cdot k_{2}}|x-1|^{k_{2} \cdot k_{3}} \\
\simeq & (-1)^{N}\left(\frac{\epsilon}{2} \sin \phi\right)^{p}\left(\frac{\epsilon}{2} \cos \phi\right)^{r}\left(-\frac{M_{1}+M_{2}}{2}\right)^{q} B\left(\frac{M_{1} M_{2}}{2}, \frac{M_{1} M_{2}}{2}\right) \\
& \cdot{ }_{2} F_{1}\left(\frac{M_{1} M_{2}}{2} ;-q ; M_{1} M_{2} ; \frac{2 M_{1}}{M_{1}+M_{2}}\right) .
\end{aligned}
$$

We are now ready to calculate the ratio of $s-t$ and $t-u$ channel amplitudes,

$$
\frac{A_{s t}^{(p, r, q)}}{A_{t u}^{(p, r, q)}}=(-1)^{N} \frac{B\left(-M_{1} M_{2}+1, \frac{M_{1} M_{2}}{2}\right)}{B\left(\frac{M_{1} M_{2}}{2}, \frac{M_{1} M_{2}}{2}\right)}
$$




$$
=(-1)^{N} \frac{\Gamma\left(M_{1} M_{2}\right) \Gamma\left(-M_{1} M_{2}+1\right)}{\Gamma\left(\frac{M_{1} M_{2}}{2}\right) \Gamma\left(-\frac{M_{1} M_{2}}{2}+1\right)} \simeq \frac{\sin \pi\left(k_{2} \cdot k_{4}\right)}{\sin \pi\left(k_{1} \cdot k_{2}\right)},
$$

where, in the nonrelativistic limit, we have,

$$
\begin{aligned}
& k_{1} \cdot k_{2} \simeq-M_{1} M_{2}, \\
& k_{2} \cdot k_{4} \simeq \frac{\left(M_{1}+M_{2}\right) M_{2}}{2} .
\end{aligned}
$$

So we have ended up with a consistent nonrelativistic level $M_{2}$ dependent string $B C J$ relations. Similar relations for $t-u$ and $s-u$ channel amplitudes can be calculated. We stress that the above relation is the stringy generalization of the massless field theory BCJ relation to the higher spin stringy particles. Moreover, as we will show now that, there exist much more relations among these amplitudes.

There existed a recurrence relation of Gauss hypergeometry function

${ }_{2} F_{1}(a ; b ; c ; z)=\frac{c-2 b+2+(b-a-1) z}{(b-1)(z-1)}{ }_{2} F_{1}(a ; b-1 ; c ; z)+\frac{b-c-1}{(b-1)(z-1)}{ }_{2} F_{1}(a ; b-2 ; c ; z)$,

which can be used to derive the recurrence relation,

$$
\begin{aligned}
\left(-\frac{M_{1}+M_{2}}{2}\right) A_{s t}^{(p, r, q)}= & \frac{M_{2}\left(M_{1} M_{2}+2 q+2\right)}{(q+1)\left(M_{2}-M_{1}\right)}\left(\frac{\epsilon}{2} \sin \phi\right)^{p-p^{\prime}}\left(\frac{\epsilon}{2} \cos \phi\right)^{p^{\prime}-p+1} A_{s t}^{\left(p^{\prime}, p+r-p^{\prime}-1, q+1\right)} \\
& +\frac{2\left(M_{1} M_{2}+q+1\right)}{(q+1)\left(M_{2}-M_{1}\right)}\left(\frac{\epsilon}{2} \sin \phi\right)^{p-p^{\prime \prime}}\left(\frac{\epsilon}{2} \cos \phi\right)^{p^{\prime \prime}-p+2} A_{s t}^{\left(p^{\prime \prime}, p+r-p^{\prime \prime}-2, q+2\right)}
\end{aligned}
$$

where $p^{\prime}$ and $p^{\prime \prime}$ are the polarization parameters of the second and third Amplitudes on the r.h.s. of eq. (4.16). For example, for a fixed mass level $N=4$, one can derive many recurrence relations for either $s-t$ channel or $t-u$ channel amplitudes with $q=0,1,2$. For say $q=2,(p, r)=(2,0),(1,1),(0,2)$. We have $p^{\prime}=0,1$ and $p^{\prime \prime}=0$. We can thus derive, for example for $(p, r)=(2,0)$ and $p^{\prime}=1$, the recurrence relation among NSS amplitudes $A_{s t}^{(2,0,2)} A_{s t}^{(1,0,3)} A_{s t}^{(0,0,4)}$ as following

$$
\left(-\frac{M_{1}+M_{2}}{2}\right) A_{s t}^{(2,0,2)}=\frac{M_{2}\left(M_{1} M_{2}+6\right)}{3\left(M_{2}-M_{1}\right)}\left(\frac{\epsilon}{2} \sin \phi\right) A_{s t}^{(1,0,3)}+\frac{2\left(M_{1} M_{2}+4\right)}{3\left(M_{2}-M_{1}\right)}\left(\frac{\epsilon}{2} \sin \phi\right)^{2} A_{s t}^{(0,0,4)} .
$$

Exactly the same relation can be obtained for $t-u$ channel amplitudes since the ${ }_{2} F_{1}(a ; b ; c ; z)$ dependence in the $s-t$ and $t-u$ channel amplitudes calculated above are the same. Moreover, we can for example replace $A_{s t}^{(2,0,2)}$ amplitude above by the corresponding $t-u$ channel amplitude $A_{t u}^{(2,0,2)}$ through eq. (4.13) and obtain

$$
\begin{aligned}
\frac{(-1)^{N}}{2 \cos \frac{\pi M_{1} M_{2}}{2}}\left(-\frac{M_{1}+M_{2}}{2}\right) A_{t u}^{(2,0,2)}= & \frac{M_{2}\left(M_{1} M_{2}+6\right)}{3\left(M_{2}-M_{1}\right)}\left(\frac{\epsilon}{2} \sin \phi\right) A_{s t}^{(1,0,3)} \\
& +\frac{2\left(M_{1} M_{2}+4\right)}{3\left(M_{2}-M_{1}\right)}\left(\frac{\epsilon}{2} \sin \phi\right)^{2} A_{s t}^{(0,0,4)},
\end{aligned}
$$

which relates higher spin NSS amplitudes in both $s-t$ and $t-u$ channels. Eq. (4.18) is one example of the extended recurrence relations in the NSS limit. For each fixed mass level $M_{2}$, 
the relation in eq. (4.18) relates amplitudes of different spin polarizations and different channels of the same propogating higher spin particle in the string spectrum. In the next subsection, we will consider a more general extended recurrence relation which relates NSS amplitudes of different higher spin particles for each fixed mass level $M_{2}$ in the string spectrum.

\subsection{More general string states}

Recently the structure of the most general NSS string amplitudes which can be expressed in terms of Gauss hypergeometry functions were pointed out [34]. Here, as an illustration, we will calculate one example of extended recurrence relation which relates NSS amplitudes of different higher spin particles for each fixed mass level $M_{2}$. In particular, the $s-t$ channel of NSS amplitudes of three tachyons and one higher spin massive string state at mass level $N=3 p_{1}+q_{1}+3$ correspond to the following three higher spin string states

$$
\begin{aligned}
& A_{1} \sim\left(i \partial^{3} X^{T}\right)^{p_{1}}\left(i \partial X^{P}\right)^{1}\left(i \partial X^{L}\right)^{q_{1}+2} \\
& A_{2} \sim\left(i \partial^{2} X^{T}\right)^{p_{1}}\left(i \partial X^{P}\right)^{2}\left(i \partial X^{L}\right)^{p_{1}+q_{1}+1} \\
& A_{3} \sim\left(i \partial X^{T}\right)^{p_{1}}\left(i \partial X^{P}\right)^{3}\left(i \partial X^{L}\right)^{2 p_{1}+q_{1}}
\end{aligned}
$$

can be calculated to be

$$
\begin{aligned}
A_{1}= & {\left[2 ! \frac{\epsilon}{2} \sin \phi\right]^{p_{1}}\left[-(1-1) ! \frac{M_{1}+M_{2}}{2}\right]^{1}\left[0 ! \frac{\epsilon}{2} \cos \phi\right]^{q_{1}+2} } \\
& \times B\left(\frac{M_{1} M_{2}}{2}, 1-M_{1} M_{2}\right){ }_{2} F_{1}\left(\frac{M_{1} M_{2}}{2},-1, M_{1} M_{2}, \frac{-2 M_{1}}{M_{1}+M_{2}}\right), \\
A_{2}= & {\left[1 ! \frac{\epsilon}{2} \sin \phi\right]^{p_{1}}\left[-(2-1) ! \frac{M_{1}+M_{2}}{2}\right]^{2}\left[0 ! \frac{\epsilon}{2} \cos \phi\right]^{p_{1}+q_{1}+1} } \\
& \times B\left(\frac{M_{1} M_{2}}{2}, 1-M_{1} M_{2}\right){ }_{2} F_{1}\left(\frac{M_{1} M_{2}}{2},-2, M_{1} M_{2}, \frac{-2 M_{1}}{M_{1}+M_{2}}\right), \\
A_{3}= & {\left[0 ! \frac{\epsilon}{2} \sin \phi\right]^{p_{1}}\left[-(3-1) ! \frac{M_{1}+M_{2}}{2}\right]^{3}\left[0 ! \frac{\epsilon}{2} \cos \phi\right]^{2 p_{1}+q_{1}} } \\
& \times B\left(\frac{M_{1} M_{2}}{2}, 1-M_{1} M_{2}\right){ }_{2} F_{1}\left(\frac{M_{1} M_{2}}{2},-3, M_{1} M_{2}, \frac{-2 M_{1}}{M_{1}+M_{2}}\right) .
\end{aligned}
$$

To apply the recurrence relation in eq. (4.15) for Gauss hypergeometry functions, we choose

$$
a=\frac{M_{1} M_{2}}{2}, b=-1, c=M_{1} M_{2}, z=\frac{-2 M_{1}}{M_{1}+M_{2}} .
$$

One can then calculate the extended recurrence relation

$$
\begin{aligned}
& 16\left(\frac{2 M_{1}}{M_{1}+M_{2}}+1\right)\left(-\frac{M_{1}+M_{2}}{2}\right)^{2}\left(\frac{\epsilon}{2} \cos \phi\right)^{2 p_{1}} A_{1} \\
& =8 \cdot 2^{P_{1}}\left(\frac{M_{1} M_{2}}{2}+2\right)\left(\frac{2 M_{1}}{M_{1}+M_{2}}+2\right)\left(-\frac{M_{1}+M_{2}}{2}\right)\left(\frac{\epsilon}{2} \cos \phi\right)^{p_{1}+1} A_{2} \\
& \quad-2^{P_{1}}\left(M_{1} M_{2}+2\right)\left(\frac{\epsilon}{2} \cos \phi\right)^{2} A_{3}
\end{aligned}
$$


where $p_{1}$ is an arbitrary integer. More extendened recurrence relations can be similarly derived.

The existence of these low energy stringy symmetries comes as a surprise from Gross's high energy symmetries [9-13] point of view. Finally, in constrast to the Regge string spacetime symmetry which was shown to be related to $\operatorname{SL}(5, C)$ of the Appell function $F_{1}[26]$, here we found that the low energy stringy symmetry is related to $\operatorname{SL}(4, C)[35]$ of the Gauss hypergeometry functions ${ }_{2} F_{1}$.

\section{Conclusion}

In this paper, we review historically two independent approaches of the four point string BCJ relation. One originates from field theory BCJ relations [3], and the other from calculation of string scattering amplitudes in the HSS limit [8]. By combining string BCJ relations with infinite linear relations of HSS amplitudes [14-16, 18-20], one obtains extended linear relations which relate HSS amplitudes of string states with different spins and different channels. Moreover, these extended linear relations can be used to reduce the number of independent HSS amplitudes from $\infty$ down to 1 [14-16, 18-20]. Similar calculation can be performed in the RSS limit [27], and one obtains extended recurrence relations in the RSS limit. These extended Regge recurrence relations again can be used to reduce the number of independent RSS amplitudes from $\infty$ down to $1[25,26]$.

We then give an explicit proof of four point string BCJ relations for all energy. We found that the equality of the string BCJ relations can be identified as the equalities of coefficients of two multi-linear polynomials of $k_{1}^{\mu}$ and $k_{3}^{\nu}$ in the $s-t$ and $t-u$ channel amplitudes. This calculation, which puts no constraints on the kinematic regimes in constrast to the previous one [3], provides an alternative proof of the one based on monodromy of integration [3] in string amplitude calculation.

Finally, we calculate both $s-t$ and $t-u$ channel NSS amplitudes of three tachyons and one higher spin string state including the leading trojectory string states at arbitrary mass levels. We discover that the mass and spin dependent nonrelativistic string BCJ relations can be expressed in terms of Gauss hypergeometry functions. As an application, we calculate examples of extended recurrence relations of low energy NSS amplitudes. For each fixed mass level $N$, these extended recurrence relations relate low energy NSS amplitudes of string states with different spins and different channels.

We believe that many string theory origins of properties of field theory amplitudes remain to be understood, and many more stringy generalizations of properties of field theory amplitudes remain to be uncovered in the near future.

\section{Acknowledgments}

We would like to thank Chung-I Tan for discussions. J.C. thanks the useful correspondence of authors of reference [4]. He also thanks Chih-Hau Fu for bringing his attention to reference [4]. This work is supported in part by the Ministry of Science and Technology and S.T. Yau center of NCTU, Taiwan. 
Open Access. This article is distributed under the terms of the Creative Commons Attribution License (CC-BY 4.0), which permits any use, distribution and reproduction in any medium, provided the original author(s) and source are credited.

\section{References}

[1] E. Witten, Perturbative gauge theory as a string theory in twistor space, Commun. Math. Phys. 252 (2004) 189 [hep-th/0312171] [INSPIRE].

[2] R. Roiban, M. Spradlin and A. Volovich, Scattering amplitudes in gauge theories, J. Phys. A 44 (2011) 450301.

[3] Z. Bern, J.J.M. Carrasco and H. Johansson, New relations for gauge-theory amplitudes, Phys. Rev. D 78 (2008) 085011 [arXiv:0805.3993] [INSPIRE].

[4] N.E.J. Bjerrum-Bohr, P.H. Damgaard and P. Vanhove, Minimal basis for gauge theory amplitudes, Phys. Rev. Lett. 103 (2009) 161602 [arXiv:0907.1425] [INSPIRE].

[5] S. Stieberger, Open \& closed vs. pure open string disk amplitudes, arXiv:0907.2211 [INSPIRE].

[6] R. Kleiss and H. Kuijf, Multi-gluon cross-sections and five jet production at hadron colliders, Nucl. Phys. B 312 (1989) 616 [inSPIRE].

[7] V. Del Duca, L.J. Dixon and F. Maltoni, New color decompositions for gauge amplitudes at tree and loop level, Nucl. Phys. B 571 (2000) 51 [hep-ph/9910563] [inSPIRE].

[8] C.-T. Chan, J.-C. Lee and Y. Yang, Notes on high energy bosonic closed string scattering amplitudes, Nucl. Phys. B 749 (2006) 280 [hep-th/0604122] [INSPIRE].

[9] D.J. Gross and P.F. Mende, The high-energy behavior of string scattering amplitudes, Phys. Lett. B 197 (1987) 129 [inSPIRE].

[10] D.J. Gross and P.F. Mende, String theory beyond the Planck scale, Nucl. Phys. B 303 (1988) 407 [inSPIRE].

[11] D.J. Gross, High-energy symmetries of string theory, Phys. Rev. Lett. 60 (1988) 1229 [INSPIRE].

[12] D.J. Gross and J.R. Ellis, Strings at superplanckian energies: in search of the string symmetry, Phil. Trans. Roy. Soc. Lond. A 329 (1989) 401.

[13] D.J. Gross and J.L. Manes, The high-energy behavior of open string scattering, Nucl. Phys. B 326 (1989) 73 [INSPIRE].

[14] C.-T. Chan and J.-C. Lee, Stringy symmetries and their high-energy limits, Phys. Lett. B 611 (2005) 193 [hep-th/0312226] [INSPIRE].

[15] J.-C. Lee, Stringy symmetries and their high-energy limit, hep-th/0303012 [INSPIRE].

[16] C.-T. Chan and J.-C. Lee, Zero norm states and high-energy symmetries of string theory, Nucl. Phys. B 690 (2004) 3 [hep-th/0401133] [InSPIRE].

[17] C.-T. Chan, P.-M. Ho and J.-C. Lee, Ward identities and high-energy scattering amplitudes in string theory, Nucl. Phys. B 708 (2005) 99 [hep-th/0410194] [INSPIRE].

[18] C.-T. Chan, P.-M. Ho, J.-C. Lee, S. Teraguchi and Y. Yang, High-energy zero-norm states and symmetries of string theory, Phys. Rev. Lett. 96 (2006) 171601 [hep-th/0505035] [INSPIRE]. 
[19] C.-T. Chan, P.-M. Ho, J.-C. Lee, S. Teraguchi and Y. Yang, Solving all 4-point correlation functions for bosonic open string theory in the high energy limit, Nucl. Phys. B 725 (2005) 352 [hep-th/0504138] [INSPIRE].

[20] C.-T. Chan, J.-C. Lee and Y. Yang, High energy scattering amplitudes of superstring theory, Nucl. Phys. B 738 (2006) 93 [hep-th/0510247] [INSPIRE].

[21] J.-C. Lee and Y. Yang, Review on high energy string scattering amplitudes and symmetries of string theory, arXiv:1510.03297 [INSPIRE].

[22] H. Kawai, D.C. Lewellen and S.-H. Henry Tye, A relation between tree amplitudes of closed and open strings, Nucl. Phys. B 269 (1986) 1 [InSPIRE].

[23] J.-C. Lee and Y. Yang, Regge closed string scattering and its implication on fixed angle closed string scattering, Phys. Lett. B 687 (2010) 84 [arXiv:1001.4843] [INSPIRE].

[24] G. Veneziano, Construction of a crossing-symmetric, Regge behaved amplitude for linearly rising trajectories, Nuovo Cim. A 57 (1968) 190 [INSPIRE].

[25] J.-C. Lee and Y. Mitsuka, Recurrence relations of Kummer functions and Regge string scattering amplitudes, JHEP 04 (2013) 082 [arXiv:1212.6915] [INSPIRE].

[26] J.-C. Lee and Y. Yang, The Appell function $F_{1}$ and Regge string scattering amplitudes, Phys. Lett. B 739 (2014) 370 [arXiv:1406.1285] [INSPIRE].

[27] S.-L. Ko, J.-C. Lee and Y. Yang, Pattens of high energy massive string scatterings in the Regge regime, JHEP 06 (2009) 028 [arXiv:0812.4190] [INSPIRE].

[28] A. Neveu and J. Scherk, Connection between Yang-Mills fields and dual models, Nucl. Phys. B 36 (1972) 155 [inSPIRE].

[29] J.-L. Gervais and A. Neveu, Feynman rules for massive gauge fields with dual diagram topology, Nucl. Phys. B 46 (1972) 381 [inSPIRE].

[30] J. Scherk, Zero-slope limit of the dual resonance model, Nucl. Phys. B 31 (1971) 222 [INSPIRE].

[31] N. Nakanishi, Remarks on Scherk's paper entitled "zero-slope limit of the dual resonance model", Prog. Theor. Phys. 48 (1972) 355 [INSPIRE].

[32] P.H. Frampton and K.C. Wali, Regge slope expansion in the dual resonance model, Phys. Rev. D 8 (1973) 1879 [InSPIRE].

[33] E. Plahte, Symmetry properties of dual tree-graph $N$-point amplitudes, Nuovo Cim. A 66 (1970) 713 [INSPIRE].

[34] S.-H. Lai, J.-C. Lee and Y. Yang, The string BCJ relations revisited and extended recurrence relations of nonrelativistic string scattering amplitudes, arXiv:1601.03813 [INSPIRE].

[35] W. Miller Jr., Lie theory and generalizations of the hypergeometric functions, SIAM J. Appl. Math. 25 (1973) 226. 